\title{
Dronedarone for the treatment of atrial fibrillation and atrial flutter: approval and efficacy
}

\author{
This article was published in the following Dove Press journal: \\ Vascular Health and Risk Management \\ 26 June 2010 \\ Number of times this article has been viewed
}

\section{Deborah Wolbrette \\ Mario Gonzalez \\ Soraya Samii \\ Javier Banchs \\ Erica Penny-Peterson \\ Gerald Naccarelli}

Penn State Heart and Vascular Institute, Penn State Milton S Hershey Medical Center, Hershey, Pennsylvania, USA

Correspondence: Deborah Wolbrette Penn State Heart and Vascular Institute, Penn State Milton S Hershey Medical Center, 500 University Drive, PO Box 850, MC H047, Hershey, Pennsylvania I7033-0850, USA

$\mathrm{Tel}+\mathrm{I} 7175313907$

Fax + I $71753 \mid 4077$

Email dwolbrette@hmc.psu.edu

\begin{abstract}
Dronedarone, a new Class III antiarrhythmic agent, has now been approved by the US Food and Drug Administration for use in patients with atrial fibrillation or atrial flutter. Approval came in March 2009 due to the positive results of the ATHENA trial showing significant reductions in all-cause mortality and cardiovascular hospitalization with dronedarone use. A post hoc analysis of the ATHENA data also suggested a decrease in stroke risk with this agent. However, due to safety concerns in the heart failure population in the earlier ANDROMEDA trial, dronedarone is not recommended for patients with an ejection fraction $<35 \%$ and recent decompensated heart failure. Dronedarone is an amiodarone analog with multichannel blocking electrophysiologic properties similar to those of amiodarone, but several structural differences. Dronedarone's lack of the iodine moiety reduces its potential for thyroid and pulmonary toxicity. Preliminary data from the DIONYSOS trial, and an indirect meta-analysis comparing amiodarone with dronedarone, showed amiodarone to be more effective in maintaining sinus rhythm, while dronedarone was associated with fewer adverse effects resulting in early termination of the drug. Dronedarone is the first antiarrhythmic drug for the treatment of atrial fibrillation and atrial flutter shown to reduce cardiovascular hospitalizations. In patients with structural heart disease who have an ejection fraction $>35 \%$ and no recent decompensated heart failure, dronedarone should be considered earlier than amiodarone in the treatment algorithm.
\end{abstract}

Keywords: dronedarone, amiodarone, atrial fibrillation, atrial flutter

\section{Introduction}

Atrial fibrillation is the most common arrhythmia requiring medical therapy, and a frequent cause of hospitalization. The number of people with atrial fibrillation in the US is projected to rise significantly in the future, reaching 16 million in $2050 .{ }^{1}$ The number of hospitalizations for atrial fibrillation has risen precipitously over the past few decades. ${ }^{2}$ Considering the morbidities associated with atrial fibrillation, including stroke and heart failure, the cost of health care for these patients is huge.

Initial management decisions for patients with atrial fibrillation include choosing rate or rhythm control, and determining stroke risk. The results of AFFIRM (Atrial Fibrillation Follow-up Investigation of sinus Rhythm Management) justified the use of rate control in older patients. The AFFIRM trial found no significant difference in mortality or stroke rate between the treatment strategies of rate or rhythm control. ${ }^{3}$ However, the mean age of patients in this study was 70 years, and the patients enrolled were fairly asymptomatic. These results may not apply to younger patients without heart disease, or to those with frequent symptoms. For these patients,

submit your manuscript | www.dovepress.com 
antiarrhythmic drug therapy may be the best approach. However, enthusiasm for antiarrhythmic drug therapy is tempered by the knowledge that this route is not without the potential risk of proarrhythmia or drug toxicity. A post hoc analysis of the AFFIRM data revealed that although maintenance of sinus rhythm was associated with better survival, this benefit was neutralized by increased mortality from antiarrhythmic drug use. ${ }^{4}$

The 2006 guidelines $^{5}$ for the management of atrial fibrillation included an algorithm for the use of antiarrhythmic drugs in the maintenance of sinus rhythm. This algorithm recommended drugs appropriate in specific cardiac disease states. The guidelines also advised inhospital initiation of drugs with significant potential for causing torsades de pointes. The older Vaughan-Williams Class IA drugs (disopyramide, quinidine, and procainamide) must be initiated in hospital because they prolong repolarization and the QT interval. They are not listed in the algorithm, and they are becoming increasingly difficult to obtain, due to drug companies having discontinued their manufacture. The Class IC agents (flecainide and propafenone) can be initiated on an outpatient basis, but in the guidelines they are only recommended for patients with lone atrial fibrillation without structural heart disease. Class III antiarrhythmic drugs (sotalol and dofetilide) can be used in patients with structural heart disease and in heart failure patients. However, due to their QT prolongation and risk of torsades de pointes, inhospital initiation is required. Since they are both cleared renally, the dose has to be adjusted according to creatinine clearance. Therefore, some patients cannot safely receive these drugs. For patients with structural heart disease who have renal disease or a prolonged baseline $\mathrm{QT}_{\mathrm{c}}$ interval, their only antiarrhythmic drug option has been amiodarone. Although this is our most effective drug for the treatment of atrial fibrillation, significant end-organ toxicities can limit its use in many patients. More treatment options have been needed to increase the quality of life in patients with symptomatic atrial fibrillation, while also decreasing morbidity and medical costs. Dronedarone, a new Class III agent, has now been approved by the Food and Drug Administration (FDA) for use in patients with atrial fibrillation.

\section{Electrophysiologic properties and pharmacokinetics}

Dronedarone is an amiodarone analog with similar multichannel blocking electrophysiologic properties. Like amiodarone, it has predominantly Class III effects, inhibiting the potassium currents $\mathrm{I}_{\mathrm{Kr}}, \mathrm{I}_{\mathrm{Ks}}, \mathrm{I}_{\mathrm{K} 1}$, and $\mathrm{I}_{\mathrm{K}-\mathrm{Ach}}$. The drug also blocks sodium and slow L-type calcium channels,${ }^{6}$ and has antiadrenergic effects. ${ }^{7}$ In spite of these similarities, the blocking effects of the two drugs are not equivalent. In vitro data show dronedarone has a stronger inhibitory effect on the peak sodium current ${ }^{8}$ and acetylcholine-activated potassium current than amiodarone. ${ }^{9}$

Dronedarone differs structurally from amiodarone in that the iodine moiety has been removed and a methane-sulfonyl group has been added. These modifications were made in an effort to reduce the end-organ adverse effects associated with amiodarone. Additionally, the methane-sulfonyl group makes dronedarone less lipophilic, greatly shortening its half-life. ${ }^{6,10}$

Based on data from clinic trials, the only recommended dose is $400 \mathrm{mg}$ twice daily. As with amiodarone, dronedarone's absorption is increased 2 to 3 times when taken with food. Steady-state plasma concentration is reached in five days, and the half-life is approximately 24 hours. There is extensive first-pass hepatic metabolism through the CYP450 system. Dronedarone is both a substrate for and an inhibitor of CYP3A4. It is also a CYP2D6 inhibitor, and can inhibit P-glycoprotein transport. Therefore, caution should be used in the setting of other drugs metabolized by these hepatic CYP450 systems. There is an almost 2-fold increase in digoxin levels and a 2- to 4-fold increase in simvastatin levels when these agents are used with dronedarone. Beta-blockers and calcium channel blockers (diltiazem and verapamil) also interact with this antiarrhythmic drug. ${ }^{11}$ Because these are all cardiovascular drugs frequently used in patients being treated for atrial fibrillation, physicians need to be aware of these interactions and adjust the doses to help prevent bradycardia or potential toxicity. Table 1 lists the main cardiovascular drugs, along with the enzyme pathway involved in their metabolism. Table 1 also outlines the resulting interaction, and the suggested dose adjustment when they are used with

Table I Cardiovascular drug interactions with dronedarone

\begin{tabular}{llll}
\hline Drug & Enzyme pathway & Effect & Dose adjustment \\
\hline Digoxin & P-glycoprotein (substrate) & 2.5-fold increase in digoxin level & Halve the digoxin dose \\
Verapamil, diltiazem & CYP3A (inhibitors) & I.4- to I.7-fold increase in dronedarone level & Lower dose of calcium channel blocker \\
Beta-blockers & CYP2D6 (substrate) & I.6-fold increase in metoprolol level & Lower beta-blocker dose \\
Simvastatin & CYP3A (substrate) & Up to 4.0-fold increase in simvastatin level & Maximum simvastatin dose 20 mg \\
\hline
\end{tabular}


dronedarone. Overall, drug interactions associated with dronedarone are minimal when compared with amiodarone. There is no significant warfarin interaction. Like amiodarone, dronedarone partially inhibits the tubular organic cation transport of creatinine, resulting in increased serum creatinine levels, despite unchanged glomerular filtration rate. ${ }^{12}$

\section{Clinical trials}

The initial dronedarone trials were DAFNE (Dronedarone Atrial FibrillatioN Study after Electrical cardioversion), EURIDIS (EURopean trial In atrial fibrillation or flutter patients receiving Dronedarone for the maIntenance of Sinus rhythm), ADONIS (American-Australian-African trial with DronedarONe In atrial fibrillation/flutter patients for the maintenance of Sinus rhythm), and ERATO (Efficacy and safety of dRonedArone for The cOntrol of ventricular rate during atrial fibrillation). These trials were designed to help establish the efficacy, dosage, and rate control with dronedarone. In DAFNE, doses of $400 \mathrm{mg}, 600 \mathrm{mg}$, or $800 \mathrm{mg}$ were given twice daily. The lowest dose was found to have the best efficacy, and was better tolerated with fewer gastrointestinal side effects. ${ }^{13}$ Based on these results, the dosage of $400 \mathrm{mg}$ twice daily was used in EURIDIS and ADONIS. ${ }^{14}$ These trials demonstrated both a significant increase in the median time to first recurrence of atrial fibrillation, and a decrease in ventricular response during atrial fibrillation recurrences. The ERATO trial further established dronedarone's effectiveness in rate control of permanent atrial fibrillation. ${ }^{15}$

ANDROMEDA (Antiarrhythmic trial with DROnedarone in Moderate to severe congestive heart failure Evaluating morbidity DecreAse) was a mortality study which was terminated early due to the dronedarone-treated group having twice the mortality rate of the placebo group. In the dronedaronetreated group, only $37 \%$ had a history of atrial fibrillation, but $62 \%$ had New York Heart Association Class III or IV congestive heart failure. The study population comprised patients hospitalized for decompensated heart failure, and the dosage used was $400 \mathrm{mg}$ twice a day. ${ }^{16}$ It has been postulated that the rise in creatinine in the dronedarone-treated group led to discontinuation of their angiotensin-converting enzyme inhibitors and other of their heart failure medicines. Although this could possibly explain the increase in mortality, an equally plausible explanation is that dronedarone worsened heart failure through a negative inotropic effect in this high risk-population. ${ }^{17}$

A large safety trial was designed to test whether dronedarone could be used in patients with atrial fibrillation and structural heart disease, ie, ATHENA (A placebo-controlled, double-blind, parallel arm Trial to assess the efficacy of dronedarone $400 \mathrm{mg}$ twice daily for the prevention of cardiovascular Hospitalization or death from any cause in patiENts with Atrial fibrillation/atrial flutter). The study enrolled patients with persistent or paroxysmal atrial fibrillation, and at least one cardiovascular risk factor. The same dose (400 mg twice daily) was used in this study as in ANDROMEDA. Patients with Class IV or recently decompensated heart failure were excluded. In contrast with the subjects in ANDROMEDA, only $4.4 \%$ of those in ATHENA had Class III heart failure. The results of ATHENA were significant reductions in the primary endpoints of all-cause mortality and cardiovascular hospitalization. The hazard ratio for the primary outcome in the dronedarone group was 0.76 . The reduced rate of hospitalizations due to cardiovascular events was mainly attributed to fewer admissions for atrial fibrillation. In the dronedarone-treated group, the only significant adverse side effects were nausea, diarrhea, bradycardia, rash, increase in serum creatinine, and QT prolongation (Table 2). The rates of thyroid and pulmonary adverse events were no different from placebo. ${ }^{18}$ Based on these positive results, dronedarone was approved by the FDA in March 2009. However, its use is limited to a lower-risk group with an EF $>35 \%$ without decompensated heart failure. Since the ATHENA population was significantly healthier than the ANDROMEDA group, the ATHENA safety data cannot be automatically applied to patients with unstable heart failure. ${ }^{17}$

A post hoc analysis of the ATHENA data was done to investigate the effect of dronedarone on stroke risk in this population. The mean $\mathrm{CHADS}_{2}$ score was 2 in both the dronedarone and placebo groups. At baseline, $60 \%$ of the patients were being treated with oral anticoagulant therapy. The risk of stroke per year was $1.2 \%$ in the dronedarone group, compared with $1.8 \%$ in the control group, with a hazard ratio of 0.66 and $P=0.027$. The reduced risk of stroke was similar, regardless of oral anticoagulant use. The relative risk of stroke was significantly decreased in

Table 2 Side effects of dronedarone*

\begin{tabular}{ll}
\hline Adverse effects & Percentage reported \\
\hline Diarrhea & $9 \%$ \\
Nausea & $5 \%$ \\
Rash & $5 \%$ \\
Bradycardia & $3 \%$ \\
Laboratory/ECG effects & \\
QT prolongation & $28 \%$ \\
Serum creatinine increase & $51 \%$ \\
\hline
\end{tabular}

Notes: "These data are based on a $400 \mathrm{mg}$ twice daily dose of dronedarone in ATHENA, EURIDES, ADONIS, ERATO, and DAFNE studies. ${ }^{13-15,18}$

Abbreviation: ECG electrocardiogram. 
patients with a $\mathrm{CHADS}_{2}$ score of $\geq 2$, compared with those with lower $\mathrm{CHADS}_{2}$ scores. ${ }^{19} \mathrm{~A}$ limitation of this analysis is that stroke was not a prespecified primary outcome of ATHENA. Therefore, the observation of decreased stroke risk in this post hoc analysis should be viewed with caution. Further studies designed to investigate this hypothesis are warranted.

In previous studies, no antiarrhythmic drugs have been found to reduce the risk of stroke in atrial fibrillation patients. Antiarrhythmic drugs were found to decrease the recurrence of atrial fibrillation in the AFFIRM trial. The lack of benefit in reducing stroke risk was thought to be most likely due to underuse of warfarin in the group treated with antiarrhythmic drugs. ${ }^{20}$ The decreased stroke risk in the dronedarone-treated group in the ATHENA trial could be attributed to reduction of atrial fibrillation frequency. Dronedarone's modest reduction of blood pressure, and more substantial decrease in heart rate during atrial fibrillation are other potential mechanisms of decreased stroke risk. ${ }^{19}$

\section{Efficacy of dronedarone}

Due to dronedarone's chemical and electrical similarities to amiodarone, it is natural to want to compare efficacy between the two drugs. Freedom from recurrent atrial fibrillation was not an endpoint in the ATHENA trial, thereby limiting information on efficacy compared with placebo in that population. Critics were disappointed that the ATHENA trial was not designed as a head-to-head comparison of dronedarone and amiodarone. However, the DIONYSOS (Efficacy and Safety of Dronedarone Versus Amiodarone for the Maintenance of Sinus Rhythm in Patients With Atrial Fibrillation) trial was conducted, ${ }^{17}$ partly to fulfill the requirements of the European Medicines Agency. This trial, comparing the efficacy of dronedarone versus amiodarone in the treatment of atrial fibrillation, has recently concluded. After a mean follow-up period of seven months, amiodarone was found to be more effective in maintaining sinus rhythm than was dronedarone. Atrial fibrillation recurrence with dronedarone was $63.5 \%$ compared with $42.0 \%$ in the amiodarone-treated group. On the other hand, dronedarone use was associated with fewer adverse effects and less premature termination of the drug. While intolerance to dronedarone was mainly secondary to gastrointestinal side effects, thyroid and neurologic events (tremor and sleep disorder) were mostly responsible for discontinuation of amiodarone. ${ }^{21}$

An indirect meta-analysis has also been published ${ }^{22}$ using 4 placebo-controlled trials of amiodarone and 4 placebo-controlled trials of dronedarone. Also included in this analysis were direct randomized data from the DIONYSOS trial. ${ }^{21}$ The authors used indirect comparison meta-analysis and normal logistic meta-regression models to compare the efficacy and safety of dronedarone with amiodarone. ${ }^{22}$ The results were similar to the data from the DIONYSOS trial alone. Dronedarone was less effective than amiodarone for the maintenance of sinus rhythm, but was associated with fewer adverse side effects necessitating discontinuation of the drug. Additionally, there was a trend toward greater all-cause mortality associated with amiodarone use. In dronedaronetreated patients, the incidence of end-organ toxicity, or symptomatic bradycardia resulting in termination of the drug, was not statistically different compared with the placebo group. However, the incidence of pulmonary and liver toxicity in the amiodarone users requiring drug discontinuation was also no different from placebo. ${ }^{22}$ Because in this analysis the average follow-up of the trials was one year and in the DIONYSOS trial the duration of follow-up was only 6 months, we do not know what the long-term difference in adverse events will be. To weigh the risks and benefits of these two drugs more accurately, more long-term follow-up data are needed.

\section{Safety and surveillance}

Although dronedarone prolongs the QT interval, the risk of torsades de pointes is low. There were no cases of torsades de pointes reported in the DIONYSOS trial, ${ }^{23}$ and only one case in the ATHENA trial. ${ }^{18}$ Similar to amiodarone, the low risk of torsades de pointes allows outpatient initiation of the drug. However, the risk of proarrhythmia could significantly increase in the setting of a $\mathrm{QT}_{\mathrm{c}}$ interval $>500 \mathrm{msec}$. Patients with a prolonged $\mathrm{QT}_{\mathrm{c}}$ were excluded from the drug trials. Dronedarone should not be used in conjunction with other drugs that prolong the QT interval, and should be used cautiously with drugs known to interact with dronedarone. Periodic electrocardiograms to monitor patients for a prolonged QT interval and bradycardia are recommended. Patients should be instructed to take dronedarone with food to increase absorption, and to avoid grapefruit juice, which can increase serum levels of this drug. Unlike amiodarone users, dronedarone patients do not need to be monitored for possible thyroid, liver, or pulmonary toxicity. Obviously, patients with severe baseline hepatic impairment should not take dronedarone, because the drug is metabolized by the CYP450 system. As with all antiarrhythmic drugs, dronedarone has not been studied in pregnant women. However, the drug has been found to be teratogenic in animal studies. Therefore, dronedarone is contraindicated 
for use during pregnancy. Due to the increased mortality in dronedarone-treated patients in the ANDROMEDA trial, there is a black box warning in the package insert against the use of the drug in patients with New York Heart Association Class IV heart failure, or Class II-III heart failure with a recent decompensation requiring hospitalization or referral to a heart failure specialist. Table 3 summarizes the contraindications to dronedarone use. These data are based on exclusion criteria from the dronedarone clinical trials. ${ }^{16,18}$

\section{Clinical role of dronedarone}

Ten years after the approval of dofetilide, the FDA approved dronedarone in 2009 to reduce the risk of cardiovascular hospitalization in patients with paroxysmal or persistent atrial fibrillation or atrial flutter. Some advantages of this new antiarrhythmic drug include outpatient initiation, as well as less need for surveillance for end-organ toxicity and fewer drug interactions. There is a single recommended dose which can be started on an outpatient basis due to the low risk of proarrhythmia. While electrocardiograms should be periodically obtained, no chest X-rays or laboratory tests to monitor thyroid and hepatic function are required. Since there is no significant interaction with warfarin, more frequent monitoring of coagulation is not needed. Dronedarone is generally well tolerated. Gastrointestinal side effects are the most common, but were responsible for discontinuation of the drug in only $3.2 \%$ of patients in clinical trials. ${ }^{24}$ These factors, along with fewer hospitalizations and decreased stroke risk seen in the ATHENA trial, ${ }^{18,19}$ may result in a decreased cost of treatment in dronedarone patients. However, no cost-efficacy analyses have been done to confirm this theory. On the less positive side, DIONYSOS and other meta-analyses have shown dronedarone to be less effective than amiodarone in preventing recurrence of atrial fibrillation. ${ }^{22,23}$ Important to remember were the safety concerns for decompensated heart failure patients in the ANDROMEDA trial. ${ }^{16}$

Although less efficacious than amiodarone in the prevention of recurrent atrial fibrillation, dronedarone reduces the risk of cardiovascular hospitalization. It is a safer, well tolerated drug in patients without decompensated heart failure. Dronedarone can be considered as an alternative therapy to amiodarone, and tried prior to amiodarone, especially in younger patients. It is also an obvious choice for patients who have developed toxicities from amiodarone. Table 4 summarizes the similarities and differences between dronedarone and amiodarone. Prior to the approval of dronedarone, the only antiarrhythmic drug alternatives for patients with structural heart disease who were intolerant to amiodarone were either sotalol or dofetilide. These drugs require inpatient initiation, and cannot be used in patients with significant renal failure. Due to the risk of torsades de pointes associated with these drugs, and the long half-life of amiodarone, physicians have typically waited a month or more to start dofetilide or sotalol after stopping amiodarone. In the ATHENA trial, ${ }^{18}$ patients had to stop amiodarone at least a month prior to enrollment in the trial. The earlier EURIDIS and ADONIS trials ${ }^{14}$ allowed patients to be enrolled immediately after discontinuation of amiodarone. Physicians are currently using their clinical judgment in deciding the optimal amiodarone washout period for each

Table 3 Indications and contraindications for dronedarone use*

Indications

To reduce the risk of cardiovascular hospitalization in patients with paroxysmal or persistent atrial fibrillation or atrial flutter

Contraindications

- NYHA Class IV heart failure, or Class II-III with recent decompensation requiring hospitalization or referral to heart failure specialist

- Second or third degree AV block or SND without a pacemaker

- Concomitant use of strong CYP 3A inhibitors (such as ketoconazole, itraconazole, voriconazole, cyclosporine, telithromycin, clarithromycin, nefazodone, and ritonavir)

- Concomitant use of QT-prolonging drugs or herbal products

- Baseline prolonged corrected QT interval (>500 msec) or PR interval (>280 msec)

- Severe hepatic dysfunction

- Women who are pregnant or may become pregnant (Category X)

Relative contraindications

- New or worsening heart failure during treatment

- Hypokalemia or hypomagnesemia

- Corrected QT interval $\geq 500$ msec on dronedarone

Notes: "Data are based on exclusion criteria for dronedarone clinical trials. ${ }^{16,18}$ Adapted from the prescribing information approved by the US Food and Drug Administration. Abbreviations: NYHA, New York Heart Association; AV, atrioventricular; SND, sinus nodal dysfunction; Category X, teratogenic in animal studies. 
Table 4 Comparison of pharmacologic and clinical characteristics of dronedarone and amiodarone

\begin{tabular}{|c|c|c|}
\hline & Amiodarone & Dronedarone \\
\hline lodine moiety & Yes & No \\
\hline Half-life & 53 days & 14-30 hours \\
\hline Blocks $I_{\mathrm{Kr}} ; I_{\mathrm{Ks}} ; \beta_{1} ; \mathrm{I}_{\mathrm{Ca}-\mathrm{L}} ; \mathrm{I}_{\mathrm{Na}} ; \mathrm{I}_{\mathrm{KI}} ; \mathrm{I}_{\mathrm{K}-\mathrm{ACh}}$ & Yes & Yes \\
\hline Dosing & Daily after loading & Twice daily (400 mg only) \\
\hline Food effect & Yes & Yes \\
\hline CYP450 3A4 metabolism & No & Yes \\
\hline Inhibits tubular secretion of creatinine & Yes & Yes \\
\hline Low torsades de pointes risk & Yes & Yes \\
\hline Outpatient initiation & Yes & Yes \\
\hline Efficacy in suppressing AF & $65 \%$ & $50 \%$ \\
\hline Efficacy in suppressing VT & Yes & Not well studied \\
\hline Decreases CV hospitalization & No & Yes \\
\hline Reduces stroke risk & No & Yes \\
\hline Warfarin interaction & Yes & No \\
\hline Pulmonary/thyroid toxicity & Yes & No \\
\hline Safety concerns in CHF & SCD-HEFT NYHA III & ANDROMEDA \\
\hline
\end{tabular}

Abbreviations: $\mathrm{I}_{\mathrm{K} \text {, }}$, rapidly activating delayed-rectifier potassium current; $\mathrm{I}_{\mathrm{Ks}}$, slowly activating delayed-rectifier potassium current; $\beta_{\mathrm{l}}$, beta-adrenergic receptors; $\mathrm{I}_{\mathrm{Ca}-\mathrm{L}}$, L-type calcium current; $I_{\mathrm{Na}}$, peak inward sodium current; $I_{\mathrm{Kl}}$, inwardly rectified potassium current; $I_{\mathrm{K}-\mathrm{ACh}}$, acetylcholine-activated potassium current; $\mathrm{CHF}$, congestive heart failure; CV, cardiovascular; VT, ventricular tachycardia; AF, atrial fibrillation; NYHA III, New York Heart Association Class III.

patient. The ARTEMIS trial has been designed to answer this question. Enrolled patients will switch from amiodarone to dronedarone either immediately, or with one week or one month delay. No one is advocating this switch in patients whose atrial fibrillation is controlled and they are not experiencing any adverse effects on amiodarone.

\section{Conclusion}

Dronedarone can be considered as a first-line therapy for atrial fibrillation or atrial flutter in patients with structural heart disease, who have an EF $>35 \%$ and no recent decompensated heart failure. Although it is less efficacious than amiodarone in maintaining sinus rhythm, dronedarone is the first drug therapy for atrial fibrillation or atrial flutter shown to reduce the rate of cardiovascular hospitalizations. This is likely due to fewer arrhythmia episodes and better tolerated atrial fibrillation recurrences. Theoretically, dronedarone use should result in lower health care cost for atrial fibrillation patients due to its favorable safety profile, lowering of stroke risk, and reduction in cardiovascular hospitalizations. Dronedarone provides an additional pharmacological option in patients with atrial fibrillation, which can be considered earlier than amiodarone in the treatment algorithm.

\section{Disclosure}

DW is a consultant/advisor for sanofi-aventis and has received research grants from Procter and Gamble, sanofi-aventis, Guidant, Medtronic, and Reliant, as well as educational grants from Boston Scientific and Medtronic. MG is a consultant/advisor for Biosense
Webster. GN is a consultant/advisor for GlaxoSmithKline, Medtronic, Boston-Scientific, Pfizer, Xention, sanofiaventis, Gilead, Novartis, Portola, AstraZeneca, BristolMyers Squibb, and Merck, and has received research grants from Boston Scientific, sanofi-aventis, and Boehringer Ingelheim. JB and SS are consultants/advisors for Boston Scientific.

\section{References}

1. Miyasaka Y, Barnes ME, Gersh BJ, et al. Secular trends in incidence of atrial fibrillation in Olmsted County, Minnesota, 1980 to 2000, and implications on the projections for future prevalence. Circulation. 2006; 114:119-125.

2. Friberg J, Buch P, Scharling H, et al. Rising rates of hospital admissions for atrial fibrillation. Epidemiology. 2003;14:666-672.

3. AFFIRM Investigators. A comparison of rate control and rhythm control in patients with atrial fibrillation. The Atrial Fibrillation Follow-Up Investigation of Rhythm Management (AFFIRM) Investigators. $N$ Engl J Med. 2002;347:1825-1833.

4. Corley SD, Epstein AE, DiMarco JP, et al. Relationships between sinus rhythm, treatment, and survival in the Atrial Fibrillation Follow-Up Investigation of Rhythm Management (AFFIRM) study. Circulation. 2004;109:1509-1513.

5. Fuster V, Ryden LE, Cannom DS, et al. ACC/AHA/ESC 2006 guidelines for the management of patients with atrial fibrillation executive summary: A report of the American College of Cardiology/American Heart Association Task Force on Practice Guidelines and the European Society of Cardiology Committee for Practice Guidelines (Writing Committee to Revise the 2001 Guidelines for the Management of Patients with Atrial Fibrillation) developed in collaboration with the European Heart Rhythm Association and the Heart Rhythm Society. J Am Coll Cardiol. 2006; 48:854-906.

6. Wegener FT, Ehrlich JR, Hohnloser SH. Dronedarone: An emerging agent with rhythm- and rate-controlling effects. J Cardiovasc Electrophysiol. 2006;17Suppl 2:S17-S20.

7. Hodeige D, Heyndrickx JP, Chatelain P, Manning A. SR33589, a new amiodarone-like anti-arrhythmic agent: Anti-adrenoceptor activity in anesthetized and conscious dogs. Eur J Pharmacol. 1995;279:25-32. 
8. Lalavee N, Nargeot J, Barrere-Lemaire S, Gautier P, Richard S. Effects of amiodarone and dronedarone on voltage-dependent sodium current in human cardiomyocytes. J Cardiovasc Electrophysiol. 2003;14: 885-890.

9. Guillemare E, Marion A, Nisato D, Gautier P. Inhibitory effects of dronedarone on muscarinic $\mathrm{K}^{+}$current in guinea pig atrial cells. J Cardiovasc Pharmacol. 2000;130:1315-1320.

10. Zareba KM. Dronedarone: A new antiarrhythmic agent. Drugs Today (Barc). 2006;42:75-86.

11. Patel C, Yan GX, Kowey PR. New drugs and technology: Dronedarone. Circulation. 2009;120:636-644.

12. Tschuppert Y, Buclin T, Rothuizen LE, et al. Effect of dronedarone on renal function in healthy subjects. Br J Clin Pharmacol. 2007; 64:785-791.

13. Touboul P, Brugada J, Capucci A, Crÿns, HJ, Edvardsson N, Hohnloser SH. Dronedarone for prevention of atrial fibrillation: A dose-ranging study. Eur Heart J. 2003;24:1481-1487.

14. Singh BN, Connolly SJ, Crÿns, HJ, et al. Dronedarone for maintenance of sinus rhythm in atrial fibrillation or flutter. N Engl J Med. 2007;357: 987-999.

15. Davy JM, Herold M, Hoglund C, et al. Dronedarone for the control of ventricular rate in permanent atrial fibrillation: The efficacy and safety of dronedarone for the control of ventricular rate during atrial fibrillation (ERATO) study. Am Heart J. 2008;156:527-529.

16. Køber L, Torp-Pedersen C, McMurray JJ, et al. Increased mortality after dronedarone therapy for severe heart failure. $N$ Engl J Med. 2008;358: 2678-2687.
17. Zimetbaum PJ. Dronedarone for atrial fibrillation - an odyssey. $N E n g l$ J Med. 2009;360:1811-1813.

18. Hohnloser SH, Crÿns, HJ, van Eickels M, et al. Effect of dronedarone on cardiovascular events in atrial fibrillation. N Engl J Med. 2009;360: 668-678.

19. Connolly SJ, Crÿns, HJ, Torp-Pedersen C, et al. Analysis of stroke in ATHENA: A placebo-controlled, double-blind, parallel-arm trial to assess the efficacy of dronedarone $400 \mathrm{mg}$ BID for the prevention of cardiovascular hospitalization or death from any cause in patients with atrial fibrillation/atrial flutter. Circulation. 2009;120:1174-1180.

20. Sherman DG, Kim SG, Boop BS, et al. Occurrence and stroke characteristics of stroke events in Atrial Fibrillation Follow-Up Investigation of sinus Rhythm Management (AFFIRM) study. Arch Intern Med. 2005;165:1185-1191.

21. Heuzey JY, De Ferrari GM, Radzik D, et al. A short-term, randomized, double-blind, parallel-group study to evaluate the efficacy and safety of dronedarone versus amiodarone in patients with persistent atrial fibrillation: The DIONYSOS study. J Cardiovasc Electrophysiol. 2010 Apr 6. [Epub ahead of print].

22. Piccini JP, Hasselblad V, Peterson ED, Washam JB, Califf RM, Kong DF. Comparative efficacy of dronedarone and amiodarone for the maintenance of sinus rhythm in patients with atrial fibrillation. J Am Coll Cardiol. 2009;54:1089-1095.
Vascular Health and Risk Management

\section{Publish your work in this journal}

Vascular Health and Risk Management is an international, peerreviewed journal of therapeutics and risk management, focusing on concise rapid reporting of clinical studies on the processes involved in the maintenance of vascular health; the monitoring, prevention and treatment of vascular disease and its sequelae; and the involvement of

\section{Dovepress}

metabolic disorders, particularly diabetes. This journal is indexed on PubMed Central and MedLine. The manuscript management system is completely online and includes a very quick and fair peer-review system, which is all easy to use. Visit http://www.dovepress.com/ testimonials.php to read real quotes from published authors. 